We think
the most
interesting
cell types
will be those
that drive the
continued
growth of each
organ

פע

DEVELOPMENTAL BIOLOGY

\title{
Single-cell RNA sequencing identifies novel kidney cell types in zebrafish
}

A new study has identified previously uncharacterized cell populations in whole-kidney marrow from zebrafish using droplet-based, single-cell RNA sequencing. As haematopoesis occurs in the kidney marrow of adult zebrafish, this work by David Langenau, Iain Drummond and colleagues provides an unbiased, comprehensive molecular analysis of kidney and blood cell types at single-cell resolution.

After verifying the validity of the single-cell transcriptomic profiling approach using transgenic zebrafish lines, the researchers isolated and sequenced whole-kidney preparations from wild-type zebrafish. They also used the technique to analyse mutant zebrafish strains that have immune cell deficiencies.

"Sequencing all cells from whole-kidney preparations allowed us to molecularly profile a wide array of cell types, including kidney nephron-specific cells as well as novel populations of kidney progenitors, vascular endothelial cells and mucin-secreting immune cells," says Drummond. These data could be useful for prioritizing genes and pathways to understand their roles in regulating each of these cell types. "We think the most interesting cell types will be those that drive the continued growth of each organ, including kidney progenitors and haematopoietic stem cells," comments Sowmya Iyer.

The researchers now plan to focus on single-cell RNA sequencing approaches that provide higher read depths, to better refine cell types and subpopulations. "We also envisage studies to spatially map the identified gene expression signatures onto the kidney stroma and marrow, providing insights into factors that regulate cell-cell interactions required for driving maintenance and self-renewal of kidney and blood stem cells," says Langenau.

Jack M. Heintze

ORIGINAL ARTICLE Tang, Q. et al. Dissecting hematopoietic and renal cell heterogeneity in adult zebrafish at single-cell resolution using RNA sequencing.J. Exp. Med. http://dx.doi.org/ 10.1084/jem.20170976 (2017) 